\title{
BASIC SCIENCE ARTICLE Phospholipase C-Gamma 2 Activity in Familial Steroid-Sensitive Nephrotic Syndrome
}

\author{
Limor Parker ${ }^{1}$, Hilla Bahat ${ }^{2}$, Michael Y. Appel ${ }^{1}$, Dina Volodarsky Baum ${ }^{3}$, Relly Forer ${ }^{3}$, Nir Pillar ${ }^{4}$, Michael Goldberg $^{1}$ and
} Michael Goldman ${ }^{2}$

BACKGROUND: Familial Steroid-sensitive Nephrotic Syndrome (SSNS) is rare, complicating the identification of candidate genes. A recent population-based approach study of SSNS identified HLA-DQA1 and Phospholipase C-Gamma 2 (PLCG2) missense coding variants as candidate loci. PLCG2 is a signaling molecule regulated by phosphorylation and is critical for $\mathrm{Ca}^{2+}$ flux in cells of the immune system.

METHODS: In order to detect a candidate gene for familial SSNS, we conducted an whole-exome sequencing in a pedigree consisting of two healthy parents, two non-identical twin brothers with SSNS, and a healthy young sibling. Flow cytometric assays were conducted to investigate the effects of the identified PLCG2 rare variants on B cell receptor-mediated PLCG2 tyrosine 759 phosphorylation, as well as on $\mathrm{Ca}^{2+}$ flux.

RESULTS: Two missense rare variants in the PLCG2 gene were detected in the affected twins. An increase in tyrosine phosphorylation of PLCG2 as well as more rapid $\mathrm{Ca}^{2+}$ flux were noted in response to stimulation in the affected twins compared to their parents.

CONCLUSIONS: Rare variants in PLCG2 segregated with disease in familial SSNS. Functional studies suggest the combined rare variants result in a gain of function in PLCG2 activity. Taken together, these results support PLCG2 as a possible candidate locus for familial SSNS.

Pediatric Research (2019) 85:719-723; https://doi.org/10.1038/s41390-018-0259-6

\section{INTRODUCTION}

Idiopathic nephrotic syndrome has a reported incidence of 1-3 cases per 100,000 children and a cumulative prevalence of 16 cases per 100,000. Idiopathic steroid-sensitive (responsive) nephrotic syndrome (SSNS) is the most common form of nephrotic syndrome in childhood. The disease outcome is favorable without progression to end-stage renal disease (ESRD). Contrary to SSNS, steroid-resistant nephrotic syndrome (SRNS) leads to ESRD in $>50 \%$ of children [1].

Familial SSNS is rare, with $3 \%$ of the patients having an affected sibling. Several initial genetic studies on familial SSNS, using either mutational analysis for known genes associated with nephrotic syndrome or a genome-wide linkage analysis, did not identify causative genes [2-4].

In 2014, Gee et al. identified mutations in epithelial membrane protein 2 (EMP2) as a monogenic cause of SSNS and SRNS disease [5]. Another recent exome array association study of SSNS on a cohort of South Asian children identified mutations in HLA-DQ and the phospholipase C-gamma 2 (PLCG2) gene, implicating them as a candidate loci [6].

PLCG2 is a signaling molecule that is integrated into the complex regulation of the immune system. It is a member of the phosphoinositide-specific phospholipase C family, is highly expressed, and is required for function of hematopoietic cells, including B cells, natural killer (NK) cells, mast cells, macrophages, and platelets [7]. PLCG2 is necessary for inositol 1,4,5-triphosphate production and subsequent Calcium $\left(\mathrm{Ca}^{2+}\right)$ release [8]. Mutations in this gene affect the activity of B cells, NK cells, and myeloid cells [9]. Mutations in PLCG2 have been associated with dominantly inherited autoinflammatory disease with immunodeficiency and cold urticaria [10]. Phosphorylation of various tyrosine residues of PLCG2 has been implicated in regulation of its lipase activity. Kim et al. showed that PLCG2 is phosphorylated on Y753, Y759, and Y1217 in response to engagement of the $B$ cell receptor in Ramos cells, as well as in murine splenic B cells [11].

In this study, we conducted a genetic evaluation of five family members, two of them being non-identical twins with SSNS, in order to detect a candidate gene for the disease. We found rare variants in PLCG2 with potential relevance to the SSNS phenotype and subsequently examined effects on protein phosphorylation and downstream $\mathrm{Ca}^{2+}$ flux following $\mathrm{B}$ cell stimulation.

\section{METHODS}

Patients

A 4-year-old Caucasian boy previously healthy, with no recurrent fever episodes or hospitalizations, and no relevant family history, presented with facial edema, hyperlipidemia, hypoalbuminemia, and urine protein/creatinine ratio in the nephrotic range after a few days of an upper respiratory tract infection. He was diagnosed

\footnotetext{
'Allergy, Immunology and Pediatric Pulmonary Institute, "Assaf-Harofeh" Medical Center, Zrifin, Israel, affilated to Sackler Faculty of Medicine, Tel Aviv University, Tel Aviv, Israel; ${ }^{2}$ Department of Pediatrics B, "Assaf-Harofeh" Medical Center, Zrifin, Israel, affilated to Sackler Faculty of Medicine, Tel Aviv University, Tel Aviv, Israel; ${ }^{3}$ Dyn Labs Ltd, "AssafHarofeh" Medical Center, Zrifin, Israel and "Variantyx Inc, Tel Aviv, Israel

Correspondence: Limor Parker (Limorparker@gmail.com)
}

Received: 2 August 2018 Revised: 21 November 2018 Accepted: 28 November 2018

Published online: 19 December 2018 


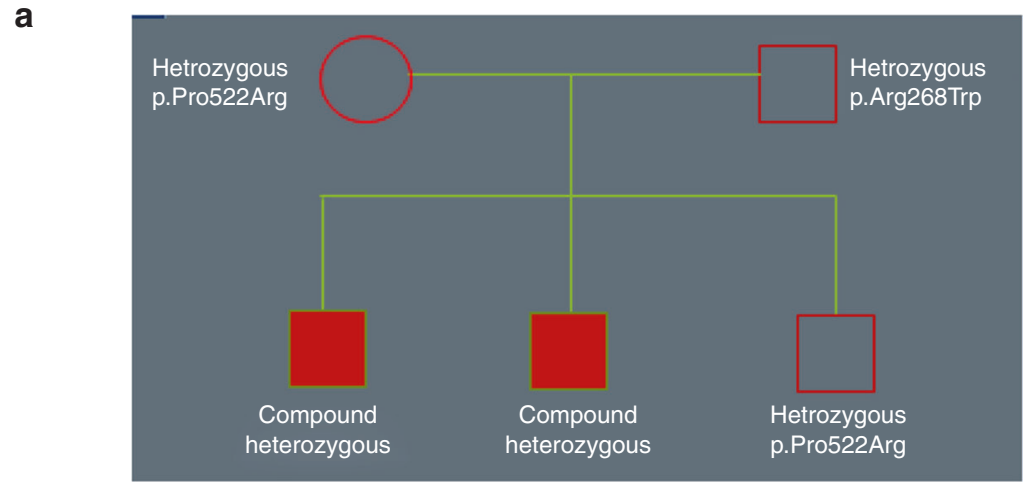

b

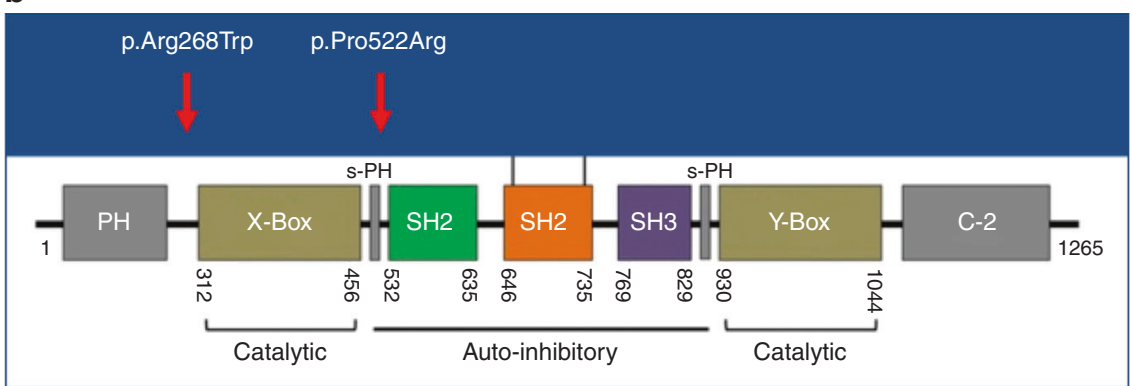

Fig. 1 Mutational analysis of the PLC-gamma 2 gene in the index family. a Pedigree of the family shows the parents as carriers of two rare variants (p.Arg268Trp in the father, p.Pro522Arg in the mother) and the two affected twins having the two variants-compound heterozygous. Sanger sequencing confirmed complete cosegregation in PLCG2 within the family. b A schematic representation of PLCG2 gene and the location of the p.Arg268Trp substitution located in the EF hand domain and the p.Pro522Arg substitution located in the auto-inhibitory (sPH) domain

with nephrotic syndrome and treatment with steroids was initiated utilizing a protocol of $60 \mathrm{mg} / \mathrm{m}^{2}$ for 6 weeks, $40 \mathrm{mg} / \mathrm{m}^{2}$ every other day for another 6 weeks, and then tapering down. He had an excellent response after a few days of treatment. In a follow-up after 4 years, no relapses were documented. One year later, his non-identical twin brother presented with similar symptoms and laboratory evaluation. No prodromal illness was documented. He was started on an identical treatment protocol with an excellent clinical response and no relapses have occurred since his original illness.

Genetic evaluation

In order to detect a candidate gene for SSNS, we conducted whole-exome sequencing (WES) from whole blood of the twins and their family members, including two healthy parents, one healthy young sibling, and the two non-identical twin siblings with SSNS. Sequencing was initially conducted by next-generation sequencing techniques, and the variations in PLCG2 were validated by Sanger sequencing. Blood samples were collected following the written informed consent under local and national ethical committee's approval. For WES, genomic DNA was extracted at Dyn Genomic Laboratory (Assaf-Harofeh, Medical Center, Israel). For validations, a separate batch of blood samples was taken from the family, and genomic DNA was extracted at Macrogen Corp. (Rockville, MD).

Whole-exome sequencing. Five samples were sent for WES using Agilent SureSelect v5 (Santa Clara, CA) and were sequenced by the Illumina HiSeq2500 at Otogenetics Inc. (Atlanta, GA). Average coverage was $98 \times$. Bioinformatics analysis and interpretation were performed by Variantyx Inc. (Framingham, MA) using Genomic Intelligence ${ }^{\circledR}$ platform. PLCG2 sequences were validated by Sanger sequencing using Applied Biosystems (ABI) (Foster City, CA) technology at Macrogen Corp. (Rockville, MD).
Evaluation of PLCG2 phosphorylation

We assayed for the phosphorylation of PLCG2 and the downstream $\mathrm{Ca}^{2+}$ flux after stimulation of B cells in peripheral blood samples from all family members. Samples (up to $6 \mathrm{ml}$ of blood in a sample) from the 5 family members were drawn for evaluation.

To identify B cells, whole blood was stained with PeCy7 antihuman CD19 (clone HIB19 (Biogems)). After 30 min, erythrocytes were lysed, and cells were washed three times with $100 \%$ fetal calf serum (FCS). Stimulation was performed in FCS with $\mathrm{F}\left(\mathrm{ab} \mathrm{b}^{\prime}\right) 2$ antihuman $\lg M+\lg G$ (eBioscience) at concentrations of 5,10 , or $50 \mu \mathrm{g} /$ $\mathrm{ml}$ for $20 \mathrm{~min}$ on ice to allow for binding and then $5 \mathrm{~min}$ in a $37^{\circ} \mathrm{C}$ water bath for activation of the intracellular cascade. Cells were immediately fixed and permeabilized, using the PerFix Expose Kit (Beckman Coulter). Intracellular staining was performed with phospho-specific (Alexa Fluor 488-conjugated mouse anti-human antibody specific for tyrosine 759 phosphorylation (Clone: K86689.37, BD Biosciences)) or its appropriately tagged isotype control (Alexa Fluor 488-conjugated mouse IgG1, kappa, (Clone: P3.6.2.8.1, eBioscience)). Following a wash, cells were assessed by flow cytometry (Navios, Beckman Coulter).

Detection of calcium release in B cells. Whole blood was stained for CD19 as described above. Following erythrocyte lysis, cells were washed in phosphate-buffered saline (PBS) solution and incubated for $30 \mathrm{~min}$ at $37{ }^{\circ} \mathrm{C}$ water bath with $100 \mathrm{nM}$ Fura Red dye (Thermo Fisher). Cells were washed twice with PBS and brought up to $500 \mu$ l with Hanks Balanced Salt Solution (Biological Industries). Cells were acquired on a flow cytometer for $140 \mathrm{~s}$ to obtain a baseline reading, then $\mathrm{F}\left(\mathrm{ab} \mathrm{b}^{\prime}\right) 2$ anti-human $\operatorname{lgM}+\lg G$ was added to a final concentration of $8 \mu \mathrm{g} / \mathrm{ml}$. The cells were then reloaded on the flow cytometer for an additional $235 \mathrm{~s}$. Calcium release was measured by calculating the ratio of Fura Red dye bound to calcium (excitation, emission: 405, $550 \mathrm{~nm}$ ) over nonbound dye (excitation, emission: 488, $670 \mathrm{~nm}$ ). Calcium release 

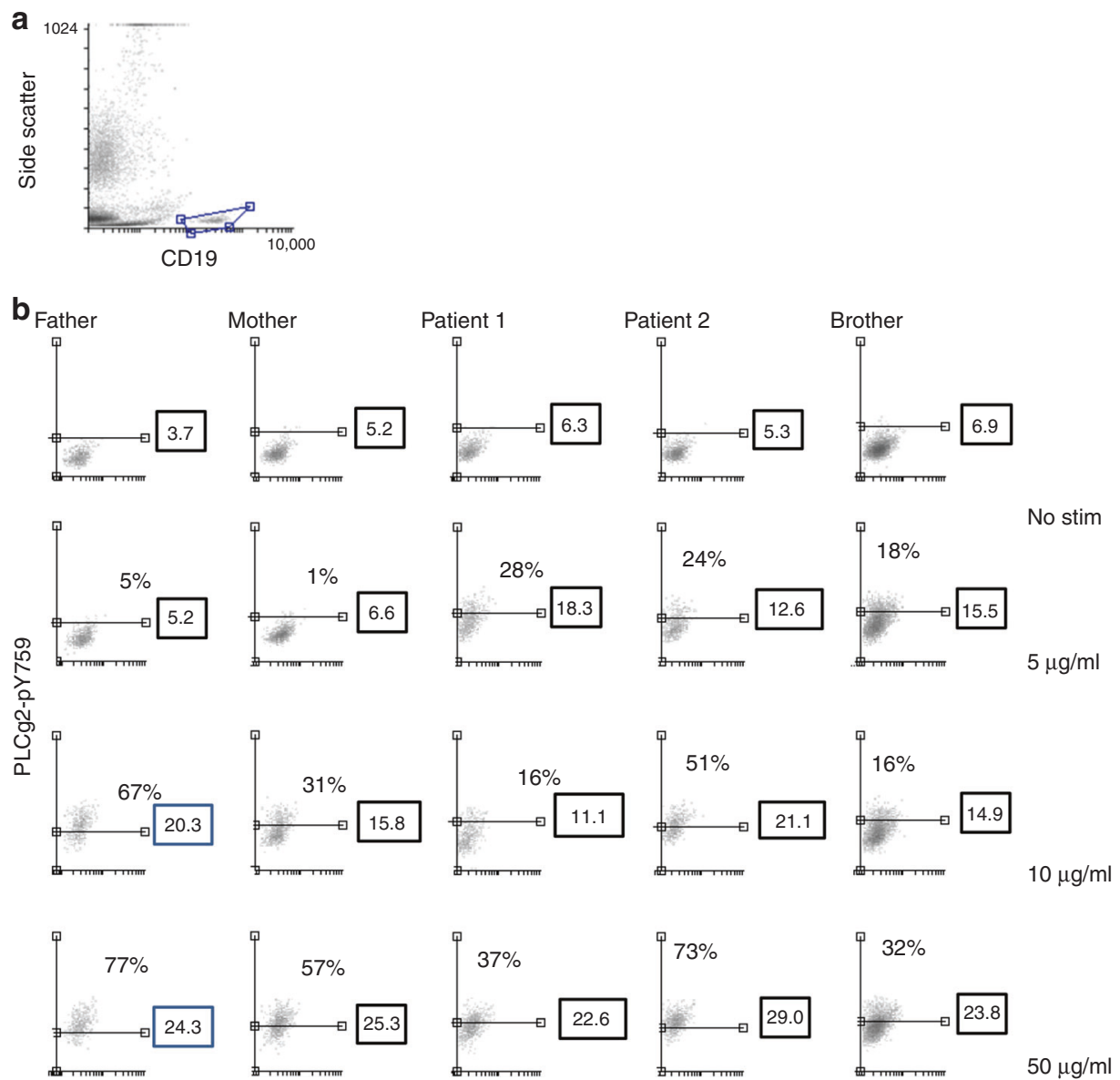

Fig. 2 Phosphorylation of PLCG2 with $5 \mu \mathrm{g} / \mathrm{ml}$ of anti-lgM/lgG. a Sample gating of CD19 ${ }^{+}$B cells as done for all subjects. b PLCG2 tyrosine 759 phosphorylation in the two brothers with SSNS compared to their parents and sibling after stimulation with anti-lgM/lgG stimulation at the different doses shown. Percentages show the CD19+ the geometric mean fluorescent intensities of Alexa Fluor 488 anti-PLCG2-pY759 staining in the CD19 ${ }^{+}$cell population

kinetics were assessed by tabulating the median bound:unbound ratios over the course of time following stimulation, for each study participant (threshold), and determining the amount of time elapsing until the greatest proportion of cells were at or above their thresholds.

\section{RESULTS}

Genetic mutational analysis

WES and Sanger sequencing yielded several mutations (Appendix 1). WES of the family generated 339,080 variants, of which 10,277 passed quality-control filters. Six hundred and sixty-two variants were located in exonic regions and 405 resulted in amino acid change. No dominant variant or homozygous recessive variants that could explain the phenotype were detected. Compound heterozygous search detected three genes (PLCG2, PLEC, and RPGRIP1L) that had maternal and paternal variant in each of the affected siblings. PLCG2 was the only gene found with reported association to SSNS that specifically segregated to the phenotype in our affected family. Two missense variants were found: a R to W in position 268 of the protein of PLCG2 (as seen in the father and the twins) and a $P$ to $R$ in position 522 of the protein (as seen in the mother and three children). (Fig. 1a). The first rare variant, PLCG2 p.Arg268Trp is a nonsynonymous single-nucleotide variation (SNV). It is located in the EF hand domain of the PLCG2 protein (Fig. 1b). It is a variant with a 0.0694 maximal allele frequency in the population databases available for review. Calculated severity score is 0.72000 on a scale of $0.0-1.0$ according to HGMD/ClinVar and 0.04 according to SIFT [12]. This variant was also found in the study of Gbadegesin et al. [6]. The second rare variant PLCG2 p.Pro522Arg is also a nonsynonymous SNV mutation. It can be found in the split pleckstrin homology $(\mathrm{sPH})$ domain of PLCG2. It is a variant with a 0.0089 maximal allele frequency in the population databases available for review. Calculated severity score is 0.22000 on a scale of $0.0-1.0$ according to HGMD/ClinVar and 0.18 according to SIFT [12] (Fig. 1b).

Both PLCG2 variants are classified as supporting pathogenicity (PP1, PP3) based on the joint consensus recommendations of the American College of Medical Genetics and Genomics and the Association for Molecular Pathology (ref. PMID: 25741868)

\section{Analysis of mutant PLCG2}

On the basis of computational predictions with HGMD/ClinVar and SIFT, we predicted that the combination of the two substitutions might render an increased function in PLCG2 signaling pathways, leading to increased PLCG2 phosphorylation, and intracellular $\mathrm{Ca}^{2}$ ${ }^{+}$release upon stimulation. In the low concentration of stimulation with anti-lgM/lgG $(5 \mu \mathrm{g} / \mathrm{ml})$, we found an increase in phosphorylated PLCG2 in the affected twins compared to their parents (Fig. 2). The healthy sibling also had an increased phosphorylation compared to the parent, although not to the same degree as the patients. Notably, the increase in phosphorylation among the affected patients was observed despite their higher baseline (unstimulated) levels of phosphorylation (Fig. 2-geometric mean fluorescent intensity values in boxes). In contrast, at $50 \mu \mathrm{g} / \mathrm{ml}$ anti$\mathrm{lgM} / \mathrm{lgG}$, phosphorylation of PLCG2 in the parents was 
Table 1. Median ratios (before and after stimulation) of bound/ unbound $\mathrm{Ca}^{2+}$ indicator in $\mathrm{CD} 19^{+}$cells and time to peak values

\begin{tabular}{llll}
\hline $\begin{array}{l}\text { Study } \\
\text { participant }\end{array}$ & $\begin{array}{l}\text { Median (Bound } \\
\text { Unbound }\end{array}$ pre & $\begin{array}{l}\text { Median (Bound } \\
\text { Unbost }\end{array}$ & $\begin{array}{l}\text { Time to }_{\text {post }} \\
\text { peak }^{\mathrm{a}}(\mathrm{s})\end{array}$ \\
\hline Patient 1 & 0.42 & 0.52 & 32 \\
Patient 2 & 0.38 & 0.57 & 32 \\
Mother & 0.37 & 0.58 & 167 \\
Father & 0.41 & 0.61 & 127 \\
Sibling & 0.43 & 0.62 & 71
\end{tabular}

aTime elapsed post stimulation until the highest percentage of cells were at or higher than the median (Bound ${ }_{\text {post }} /$ Unbound $_{\text {post }}$ ) value

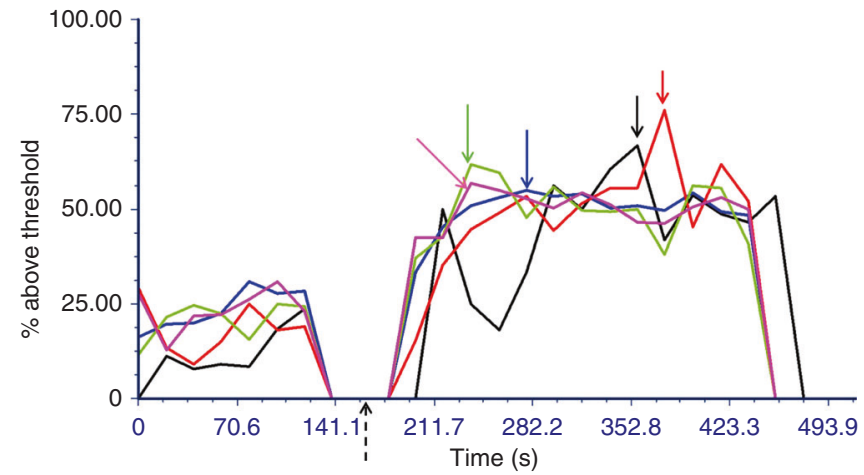

Fig. 3 Depiction of $\mathrm{Ca}^{2+}$ release after $\operatorname{lgM} / \operatorname{lgG}$ stimulation of $\mathrm{B}$ cells. Median (threshold) ratios of calcium-bound Fura Red:unbound Fura Red in $\mathrm{CD} 19^{+}$cells following stimulation were calculated for each participant. The percentage of cells with ratios at or above their thresholds are plotted over time. Dashed arrow denotes addition of stimulation. Light green line: patient 1, pink line: patient 2, red line: mother, black line: father, blue line: brother. Colored arrows denote the peaks for each respective participant. The post-stimulation reading for the father commenced approximately $25 \mathrm{~s}$ later than for other participants

comparable or higher in relation to the affected twins. While $\mathrm{Ca}^{2+}$ release in stimulated $B$ cells was slightly lower in the two patients compared to the parents and the healthy sibling (Table 1), the release kinetics were notably more rapid in the patients than in their parents (Table 1 and Fig. 3).

\section{DISCUSSION}

In this study, using WES to identify candidate loci for familial nephrotic syndrome, we observed that rare variants in PLCG2 segregated with disease, which was further validated by Sanger sequencing. Most prior genetic studies on familial SSNS using mutational analysis for known genes associated with nephrotic syndrome or genome-wide linkage analysis did not identify causative genes [2-4]. In 2014, Gee et al. identified mutations in EMP2 as a monogenic cause of SSNS and SRNS disease [5].

In a landmark study identifying risk alleles for nephrotic syndrome, an exome array association analysis of a cohort of South Asian children identified HLA-DQA1 as a candidate locus [6]. The HLA-DQA1 region, however, appears to be pleotropic for different glomerular diseases because other variants in the gene have been associated with $\lg A$ and membranous nephropathy $[13$, 14]. Additionally, this locus explained only about $4.6 \%$ of the risk for SSNS, suggesting that there are other risk variants. In the same study utilizing a rare variant analysis, mutations in the gene encoding PLCG2 were found to be associated with SSNS [6].
Our identification of PLCG2, including one of the same variants described in the aforementioned study segregating with familial SSNS in a distinct ethnic and geographical background, bolsters this gene as a potential candidate locus. The combinatorial effects of both missense mutations found in this study resulted in a net increase in PLCG2 activity as evidenced by increased phosphorylation of a tyrosine residue on PLCG2 associated with activation and downstream effects on the kinetics of calcium flux in cells of these patients. This is the first description of such changes in patients with SSNS and provides causal, albeit preliminary, evidence implicating PLCG2 in SSNS.

The first rare variant, PLCG2 p.Arg268Trp is one of the six mutations identified by Gbadegesin et al. and is located in the EF hand domain of the protein. This domain is critical for calcium binding and additionally has been implicated in the binding of the $\mathrm{PH}$ domain to phosphoinositides $[15,16]$. The second variant PLCG2 p.Pro522Arg is found in the sPH domain, which is part of the auto-inhibitory component of the PLCG2 gene, normally coupling the enzymatic activity of PLCG2 to upstream pathways [17]. We demonstrate that these variants are associated with a gain of function for phospholipase activity, as assessed by a flow cytometry-based phosphorylation assay. Furthermore, it was noted that while overall $\mathrm{Ca}^{2+}$ release in response to $\mathrm{B}$ cell stimulation was slightly lower in the patients with the two PLCG2 mutations compared to the rest of their family, their $\mathrm{Ca}^{2+}$ release kinetics were more rapid.

Mutations described in PLCG2 have been associated clinically with both auto-immune disease and immunodeficiency with dominant patterns of inheritance [9]. In a mouse model, it was demonstrated that a gain-of-function mutation in PLCG2 resulted in auto-activation of B cells and innate immune cells [18]. Similarly, the PLCG2 rare variants found in the family in our study would be classified as yielding a gain of protein function, normally resulting in a dominant inheritance pattern. It is therefore noteworthy that we and others observed the SSNS phenotype to be recessive. One possibility is that the two variants may each partially contribute to a phenotype of auto-activation. It may also be that the effects of a hyperactive form of PLCG2 could be tempered by other proteins in the calcium release pathway. Alternatively, many immunerelated cell types consist of regulatory sublineages (e.g., regulatory $T$ cells or regulatory B cells) in which a PLCG2 gain of function could mitigate the response of hyperactivated cells. The roles of PLCG2 mutations in SSNS must therefore be considered in the broader complex nature of the disease.

The study is limited by the small number of patients, the difficulty to control the effects of ageing on B cell function, and the inability to obtain multiple blood samples from the family, particularly during episodes of active disease, as opposed to remission. Furthermore, while we limited our study to B cells, other adaptive and innate immune cells express PLCG2 and could be considered. Finally, future experimentation utilizing gene constructs with the familial mutations and comparing the results with control samples without the two mutations will allow further study of their functional roles.

In conclusion, we identified rare variants in PLCG2 segregating with disease in familial SSNS. The effects of these variants result in a gain of function of PLCG2 activity. The effects of these mutant forms of PLCG2 in familial SSNS may illustrate vital elements of phospholipase-mediated signaling, including its role in leukocyte function and host defense. More research is required to evaluate the specific role and impact of this gene on the disease.

\section{ACKNOWLEDGEMENTS}

This work was supported by the Assaf-Harofeh Medical Center (Israel) "Diamond Project," a clinical and research developmental award to L.P. M.G. is funded by a Kamea grant from the Ministry of Health, Israel. The funding sources played no role in the writing of the manuscript or the decision to submit it for publication. 


\section{ADDITIONAL INFORMATION}

The online version of this article (https://doi.org/10.1038/s41390-018-0259-6) contains supplementary material, which is available to authorized users.

Competing interests: The authors declare no competing interests.

Publisher's note: Springer Nature remains neutral with regard to jurisdictional claims in published maps and institutional affiliations.

\section{REFERENCES}

1. Kang, H. G. Treatment of steroid-resistant pediatric nephrotic syndrome. Korean J. Pediatr. 54, 317-21 (2011).

2. Xia, Y. et al. Familial steroid-sensitive idiopathic nephrotic syndrome: seven cases from three families in China. Clinics (Sao Paulo) 68, 628-31 (2013).

3. Fuchshuber, A. et al. Clinical and genetic evaluation of familial steroid-responsive nephrotic syndrome in childhood. J. Am. Soc. Nephrol. 12, 374-8 (2001).

4. Landau, D. et al. Familial steroid-sensitive nephrotic syndrome in Southern Israel: clinical and genetic observations. Pediatr. Nephrol. 22, 661-9 (2007).

5. Gee, H. Y. et al. Mutations in EMP2 cause childhood-onset nephrotic syndrome. Am. J. Hum. Genet. 94, 884-90 (2014).

6. Gbadegesin, R. A. et al. HLA-DQA1 and PLCG2 are candidate risk loci for childhood-onset steroid-sensitive nephrotic syndrome. J. Am. Soc. Nephrol. 26, 1701-10 (2015)

7. Hiller, G. \& Sundler, R. Regulation of phospholipase $C$ gamma 2 via phosphatidylinositol 3-kinase in macrophages. Cell. Signal. 14, 169-73 (2002).

8. Chiang, C. Y., Veckman, V., Limmer, K. \& David, M. Phospholipase C gamma 2 and intracellular calcium are required for LPS-induced Toll Receptor 4 (TLR4) endocytosis and Interferon Regulatory Factor 3 (IRF3) activation. J. Biol. Chem. 287, 3704-9 (2012). 3.

9. Zhou, Q. et al. A hypermorphic missense mutation in PLCG2, encoding phospholipase $\mathrm{Cg} 2$, causes a dominantly inherited autoinflammatory disease with immunodeficiency. Am. J. Hum. Genet. 91, 713-20 (2012).

10. Ombrello, M. J. et al. Cold urticaria, immunodeficiency, and autoimmunity related to PLCG2 deletions. N. Engl. J. Med. 366, 330-8 (2012).

11. Kim, Y. J., Sekiya, F., Poulin, B., Bae, Y. S. \& Rhee, S. G. Mechanism of B-cell receptor-induced phosphorylation and activation of phospholipase C-gamma2. Mol. Cell. Biol. 24, 9986-99 (2004).

12. Kumar, P., Hneikoff, S. \& Ng, P. C. Predicting the effects of coding non synonymous variants on protein function using the SIFT algorithm. Nat. Protoc. 4, 1073-81 (2009).

13. Konrad, M. et al. HLA class 2 associations with idiopathic nephrotic syndrome in children. Tissue Antigens 43, 275-80 (1994).

14. Alana, M. \& Rashed, A. Genetics of childhood steroid sensitive nephrotic syndrome. Pediatr. Nephrol. 32, 1481-8 (2017).

15. Yamamoto, T.-a. et al. Involvement of EF hand motifs in the Ca2+-dependent binding of the pleckstrin homology domain to phosphoinositides. Eur. J. Biochem. 265, 481-90 (1999).

16. Hagen, $\mathrm{S}$. et al. The $\mathrm{B}$ cell receptor-induced calcium flux involves a calcium mediated positive feedback loop. Cell Calcium 51, 411-7 (2012).

17. Georgina, E., Rebecca, S. \& Julie, W. Molecular dynamic modeling of a novel PLCG2 variant reveals key protein structural differences. Alzheimers Dement. 13, 1489-90 (2017).

18. $\mathrm{Yu}, \mathrm{P}$. et al. Autoimmunity and inflammation due to a gain-of-function mutation in phospholipase $\mathrm{C}_{2} 2$ that specifically increases external Ca2+ entry. Immunity 22, 451-65 (2005). 